\title{
In-Situ Analytical Transmission Electron Microscopy Study of Electrochemical Lithiation of a Sulfur - Carbon Nanotube Composite Cathode
}

\author{
Jeremy Ticey ${ }^{1}$, Vladimir Oleshko ${ }^{1,3}$, Yujie Zhu ${ }^{2}$, Chunsheng Wang ${ }^{2}$, and John Cumings ${ }^{1}$ \\ 1. Department of Materials Science and Engineering, University of Maryland, College Park, MD \\ 2. Department of Chemical and Biomolecular Engineering, University of Maryland, College Park, MD \\ 3. Materials Science and Engineering Division, Material Measurement Laboratory, National Institute of \\ Standards and Technology, Gaithersburg, MD
}

Rechargeable Li-S batteries have the potential to meet the high power demands of next generation lightweight, low-cost, and environmentally friendly batteries useful in both small-scale portable devices and large-scale applications such as electric vehicles. Lithium-sulfur (Li-S) batteries offer a high theoretical capacity of $1,672 \mathrm{mAh} / \mathrm{g}$ and a theoretical energy density of $2567 \mathrm{Wh} / \mathrm{kg}$, roughly five times larger than that of currently utilized carbon-based Li-ion batteries. [1] In addition, sulfur is light weight, earthly abundant, and nontoxic. Despite its promise, Li-S batteries still suffer from poor cycling performance caused by the polysulfide shuttle process which occurs during the multistep reduction of sulfur to $\mathrm{Li}_{2} \mathrm{~S}$. Due to the insulating nature of sulfur, this reaction relies on the incorporation of sulfur into a conductive carbon host structure. Various carbons serve as both an electrically conductive pathway, as well as a structural network to accommodate the volumetric expansions associated with cycling. Carbon nanotubes (CNTs), possessing a high electrical conductivity and mechanical strength, are considered a prospective material to serve this role. In spite of extensive efforts, significant gaps still remain in the understanding of the behavior of lithium and reduced sulfur species at the carbon-sulfur interface during working conditions. Therefore a deeper understanding of the fundamental electrochemical reaction mechanisms and kinetics of this system is required to develop the next generation of ultrafast, long-life, high-energy density Li-S batteries. [2]

We employ in-situ transmission electron microscopy (TEM) which offers the opportunity to investigate complex reactions in real time while obtaining valuable information on the structural, chemical, and electrical properties during these processes. Using energy-filtering TEM (EFTEM) and scanning TEM (STEM) imaging modes, as well as various analytical methods, such as electron energy loss spectroscopy (EELS), energy-dispersive X-ray spectroscopy (EDXS), and electron diffraction, we have monitored the evolution of these complex structures starting from pristine composites (FIG. 1). Our insitu work utilizes a customized TEM sample holder. The holder has a small piece of metallic lithium attached to a movable piezo-driven STM tip, which served as the anode and reference electrode. A thin layer of $\mathrm{Li}_{2} \mathrm{O}$ formed during loading, was used as a solid electrolyte. The $\mathrm{Li} / \mathrm{Li}_{2} \mathrm{O}$ tip is then contacted with the S-CNT cathode and cycled through the application of an external bias. In-situ observations indicate complex morphological and compositional changes of both the Li anode and S-CNT cathode during cycling. (FIG. 2)

\section{References:}

[1] P.G. Bruce, et al, Nature Materials 11 (2012), p. 19.

[2] V.P. Oleshko, et al, Microsc Microanal 15 (2009), p. 1398. 

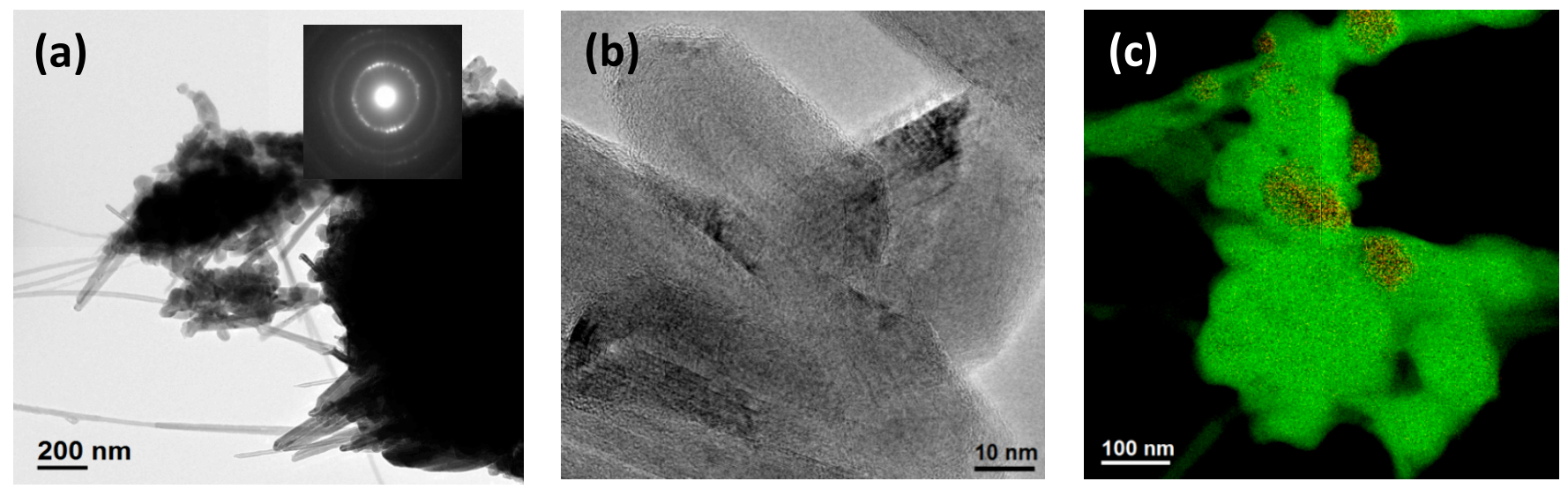

Figure 1. (a) Zero-loss energy filtered TEM image of a thick pristine S-MWCNT composite and corresponding SAED pattern of the composite (right inset). (b) HRTEM image showing nanoscale morphologies of the S-MWCNT composites. (c) Overlaid electron spectroscopic S $\mathrm{L}_{2,3}$ - map (orange) and C K- map (green) of the S-MWCNT composites

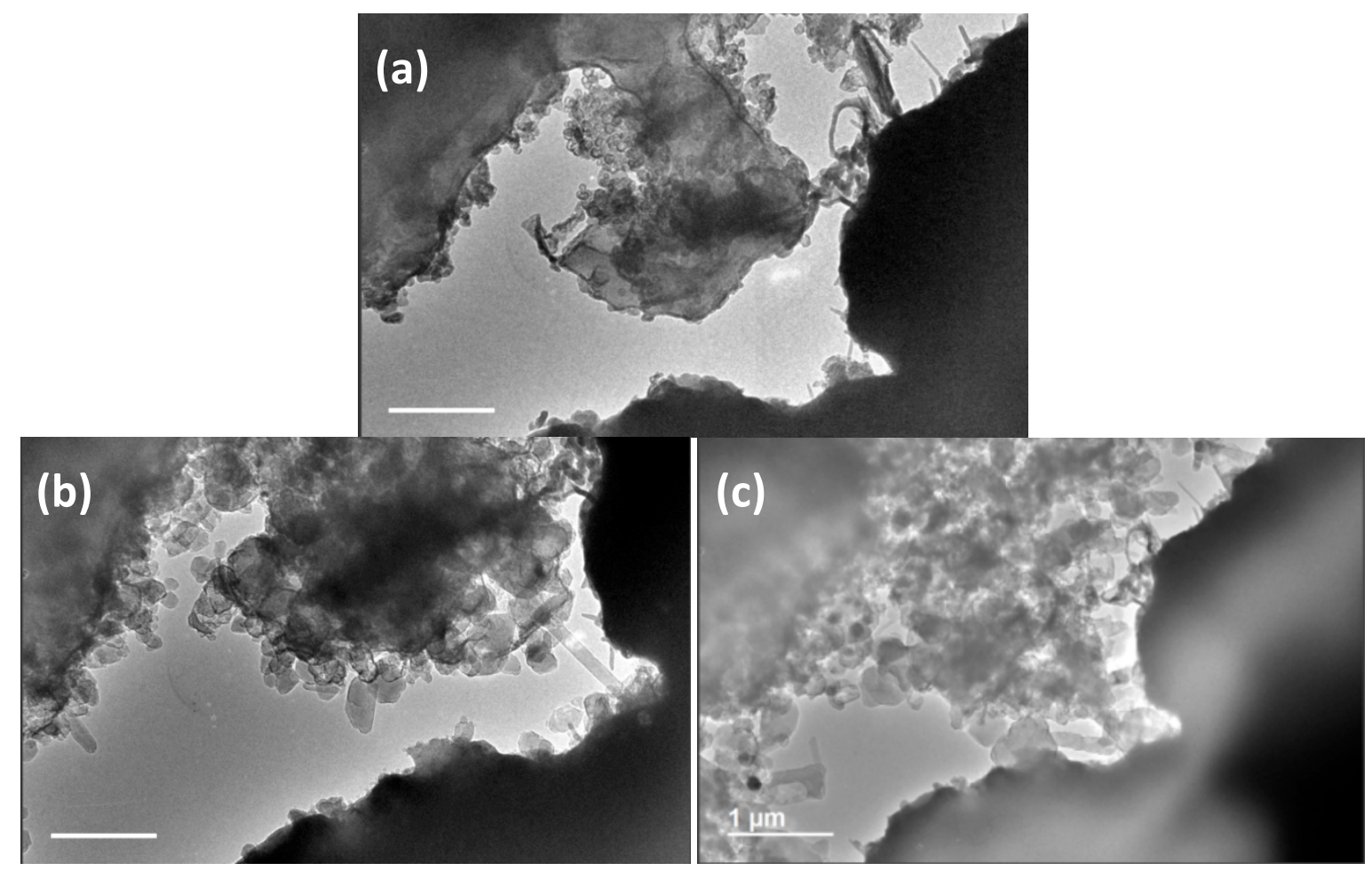

Figure 2. In situ TEM frames acquired during lithiation of S-MWCNT composites at negative $3 \mathrm{~V}$ relative to the $\mathrm{Li}$ electrode. (a) after initial contact of $\mathrm{Li} / \mathrm{Li}_{2} \mathrm{O}$ (left) and $\mathrm{S}-\mathrm{MWCNT}$ composite (right); (b) after 50 minutes of discharge; (c) after 100 minutes of discharge. All scale bars are $1 \mu \mathrm{m}$ in length. 